\title{
A Retrospective Cohort Study Comparing In-Person and Telemedicine-Based Opioid Agonist Treatment in Ontario, Canada, Using Administrative Health Data
}

\author{
Kristen A. Morin ${ }^{a}$ Matthiew D. Parrotta ${ }^{a}$ Joseph K. Eibl ${ }^{b}$ David C. Marsh ${ }^{a}$ b, c, d \\ aNorthern Ontario School of Medicine Sudbury, Sudbury, ON, Canada; ${ }^{b} \mathrm{Health}$ Sciences North Research Institute, \\ Sudbury, ON, Canada; 'ICES North, Sudbury, ON, Canada; 'Canadian Addiction Treatment Centres, Markham, ON, \\ Canada
}

\author{
Keywords \\ Canada - Epidemiology · Mortality · Opioid maintenance · \\ Telemedicine
}

\begin{abstract}
Background: This study evaluated how telemedicine as a modality for opioid agonist treatment compares to in-person care. Methods: We conducted a retrospective cohort study of patients enrolled in opioid agonist treatment between January 1, 2011, and December 31, 2015, in Ontario, Canada. We compared patients who received opioid agonist treatment predominantly in person, mixed, and predominantly by telemedicine. We used a logistic regression model to evaluate mortality, a Cox proportional hazard model to assess retention, and a negative binomial regression model to evaluate emergency department visits and hospitalizations. The study was performed using administrative health data with physician billing data from the Ontario Health Insurance Plan and prescription data from the Ontario Drug Benefit databases. Results: A total of 55,924 individuals were included in the study. Receiving opioid agonist treatment by predominantly telemedicine was not associated with all-
\end{abstract}

cause mortality $(\mathrm{OR}=0.9,95 \% \mathrm{Cl}: 0.8-1.0), 1$-year treatment retention $(\mathrm{OR}=1.0,95 \% \mathrm{Cl}: 0.9-1.1)$, or opioid-related emergency department visits and hospitalizations when compared to in-person care. The rate of emergency department visits (IRR $=1.4)$, the rate of mental health-related emergency department visits (IRR $=1.5)$, and the rate of mental healthrelated hospitalizations per year (IRR $=1.2$ ) was higher for patients who received opioid agonist treatment predominantly by telemedicine compared to in person. Conclusion: Our findings support the conclusion that telemedicine is equal to in-person care regarding mortality opioid-related emergency department visits and retention, and is a viable option for those seeking opioid agonist treatment.

$$
\begin{aligned}
& \text { (c) } 2021 \text { The Author(s) } \\
& \text { Published by S. Karger AG, Basel }
\end{aligned}
$$

\section{Introduction}

Opioid use is a topic of concern across North America, devastating communities and creating a strain on the health care systems [1-4]. In Canada, the gold standard of treatment for opioid use disorder is opioid agonist treatment (OAT), including methadone and buprenor-
(C) 2021 The Author(s)

Published by S. Karger AG, Basel

This is an Open Access article licensed under the Creative Commons Attribution-NonCommercial-4.0 International License (CC BY-NC) (http://www.karger.com/Services/OpenAccessLicense), applicable to the online version of the article only. Usage and distribution for commercial purposes requires written permission.
David C. Marsh

Northern Ontario School of Medicine

935 Ramsey Lake Rd.

Sudbury, ON P3E 2C6 (Canada)

dmarsh@nosm.ca 
phine/naloxone $[5,6]$. OAT works by alleviating withdrawal symptoms to help patients stabilize physically, mentally, and socially [7]. In Ontario, most patients will begin receiving treatment in specialized OAT clinics. At the time of this study (in Ontario), only physicians could prescribe methadone, and a nurse or pharmacist could supervise observed daily dosing during treatment stabilization [8-10]. In rural and northern communities, this can be especially difficult as the distance to physicians is greater [11], and the availability of clinics and primary health care providers is lower [12].

As part of the Ontario Government's strategy to improve access to specialized care for patients, the Ontario Telemedicine Network provided a secure telemedicine infrastructure to deliver health care when appropriate for any type of therapeutic area of care, including OAT [13]. Telemedicine services allow for remotely located physicians to interact with patients in videoconferencing sites, using local telemedicine-enabled clinics where the patients are hosted. Under the supervision of a registered nurse, locally, all OAT-related practice guidelines (including length and frequency of visits) are employed consistent with in-person treatment. Many OAT physicians in Ontario have a mix of in-person and telemedicine patients. Telemedicine has become widely utilized in addiction medicine. It has greatly increased access to care in typically underserviced areas of Ontario [13-15].

A previous work by Eibl et al. [16] used an in-clinic electronic health record to assess the effectiveness of telemedicine-delivered care with the focus on OAT retention and urine drug screening for the period of 2010-2012. Eibl et al. [16] demonstrated that telemedicine was associated with a higher likelihood of treatment retention and reduced opioid use. However, the study dataset was unable to examine the broader health outcomes related to telemedicine versus in-person care. Thus, it was critical to evaluate whether patients receiving telemedicine-delivered OAT have similar health system outcomes (including patient hospitalizations, emergency department visits, and mortality) to patients treated in person. Our objective was to examine broad health outcomes associated with telemedicine-delivered OAT compared to inperson OAT for patients in Ontario.

\section{Methods}

\section{Study Design and Overview}

We conducted a retrospective cohort study of patients initiating OAT using administrative health data between January 1, 2011, and December 31, 2015, in Ontario, Canada. Every patient in the study was followed from their first OAT episode for 1 year. If patients started OAT at the end of 2015, they were followed until December 31, 2016. Anonymized individual-level data records collected from Ontario's publicly funded health services were extracted by ICES (Institute for Clinical Evaluative Sciences) and accessed remotely for analysis. ICES is a not-for-profit research organization that gathers population-based health and social data from Ontario's publicly funded health services to generate knowledge [17]. These data were the most appropriate to answer our research question since they allowed us to evaluate populationlevel outcomes to understand the impact of interventions across health care settings over time. This study is reported following the STROBE guidelines [18] and approved by the Research Ethics Board of Laurentian University in Sudbury.

\section{Study Population: First-Time OAT Patients}

The primary cohort was created by identifying OAT patients with the Ontario Drug Benefit Plan (ODB) database using drug identification numbers and with the Ontario Health Insurance Plan (OHIP) database physician billing codes including OAT monthly management codes (K682, K683, and K684), visit/consultation codes (A680 and A957), and point of care testing codes (G040, G041, G042, or G043). The study included all patients initiating OAT for the first time within the study time frame in Ontario. First-time OAT was defined as no previous history of treatment in the year before the first treatment episode. It is common for OAT patients to cycle between treatment and relapse. Studies have demonstrated that multiple treatment attempts are correlated with a higher likelihood of positive outcomes [19-21]. We chose only to include first-time OAT patients to eliminate bias related to numerous treatment attempts.

All patients were at least 15 years or older. Other important publications have used similar age groups because of the high number of youth involved in OAT and the prevalence of adverse events in younger age groups [22]. For instance, in a report by Gomes et al. [23], the authors found that almost $60 \%$ of accidental deaths occurred among youth and younger adults (15-44 years) in Ontario [23]. In Ontario, methadone used for addiction treatment is dispensed exclusively in liquid formulation. Therefore, patients with over $20 \%$ of their methadone dose in tablet formulation over 1 year were excluded due to the likelihood that methadone was being administered for chronic pain. We also excluded patients who were not eligible for OHIP, non-Ontario residents, and those with missing age, gender, and postal codes. We then combined patients identified from ODB, patients identified from OHIP, and patients who were identified in both databases to create the primary study cohort. See Figure 1.

\section{Data Sources}

ICES administrative data were accessed remotely using a secure server. Patient information was linked anonymously across databases using encrypted 10-digit health card numbers. The linking protocol is used routinely for health system research in Ontario [24-26].

All diagnosis information from physician visits was determined using billing data from the Ontario Health Insurance Plan (OHIP) database. Emergency department visits were identified using the Canadian Institute for Health Information National Ambulatory Care Reporting System. Hospital admissions were identified using the Canadian Institute for Health Information Dis- 
Fig. 1. Data linkages. OAT, opioid agonist treatment.

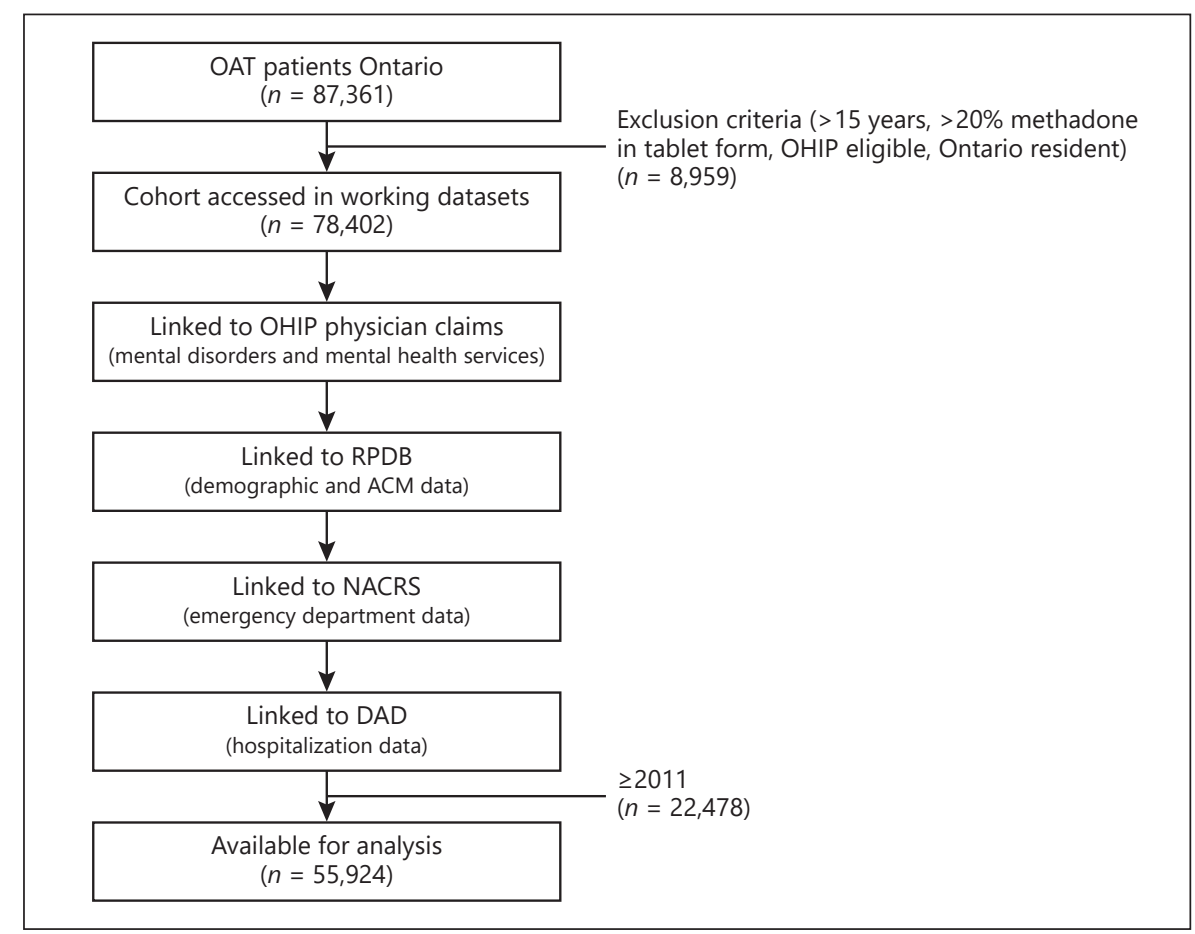

charge Abstract Database. We obtained patients' location of residence and demographic information, including all-cause mortality, from the Ontario Registered Persons Database, which contains unique data for each resident who has ever received insured health. See Figure 1. All data relating to retention in OAT (including data for methadone and buprenorphine/naloxone) were extracted from the ODB database.

\section{Exposure (Telemedicine)}

The OHIP database was used to identify telemedicine billing information, including the following OHIP fee codes: B100, B101, B102, B200, B201, and B202. Patients were assigned to one of the 3 groups: predominantly in person ( $0-25 \%$ telemedicine usage), mixed (26-75\%), and predominantly telemedicine (76-100\%). The classification of groups was decided based on the bimodal distribution of the OHIP billing data. Most patients received OAT services via in person or telemedicine, and very few patients received all of their OAT services via one modality over another. For instance, patients who would regularly receive in-person care may connect with their physician via telemedicine on rare occasion and vice versa.

\section{Covariates}

The covariates available for the study included age, sex, location of residence, income quintile, HIV, and deep tissue infection including endocarditis (OHIP fee code 429), osteomyelitis (OHIP fee code 730), and septic arthritis (OHIP fee code 711). The covariates were selected strategically based on factors identified by our research team's Patient and Family Advisory Group, the WHO Social Determinants of Health Framework, and the data variables available in the dataset used for the study.

\section{Outcomes}

All-Cause Mortality

The Registered Persons Database was used to identify all-cause mortality. All-cause mortality was linked by a patient identifier for the analysis. The all-cause mortality variable allowed us to avoid omitting deaths from causes other than opioid overdose that are important to consider for this population, such as suicide, endocarditis, and accidental deaths.

\section{Emergency Department Visits}

The National Ambulatory Care Reporting System (NACRS) database was used to identify emergency department visits. Emergency department visits were counted if an event appeared after an index date in a publicly funded Ontario hospital. A diagnostic code accompanied each emergency department event in the NACRS database. Diagnostic codes were used to categorize types of emergency department visits. Opioid-related, mental health-related, and reasons other than mental health or opioids were included in the analysis.

\section{Hospitalizations}

The Discharge Abstract Database (DAD) was used to identify hospitalizations. Hospitalizations were counted if a hospitalization discharge record appeared after a patient's index date in a publicly funded Ontario hospital. Hospitalizations were captured in 3 groups: opioid-related, mental health-related, and for reasons other than mental health or opioids, measured and coded in the same manner. Categories were specified using OHIP fee codes. Emergency department visits and hospitalizations have become a priority for policymakers in Ontario and across many health systems. We chose to report on mental health and opioid use disorder specific acute care use to align with health system quality performance metrics used in Ontario [27]. 
Table 1. Patient group characteristics

\begin{tabular}{|c|c|c|c|c|}
\hline $\begin{array}{l}\text { Variable } \\
N=45,809\end{array}$ & $\begin{array}{l}\text { Predominantly } \\
\text { in person } \\
41,798(74.7)\end{array}$ & $\begin{array}{l}\text { Mixed } \\
6,067(10.9)\end{array}$ & $\begin{array}{l}\text { Predominantly } \\
\text { telemedicine } \\
8,059(14.4)\end{array}$ & $d$ \\
\hline \multicolumn{5}{|l|}{ Age } \\
\hline $15-24$ & $7,069(69.0)$ & $1,405(13.7)$ & $1,770(17.3)$ & 9.1 \\
\hline $25-34$ & $13,762(72.4)$ & $2,372(12.5)$ & $2,872(15.11)$ & \\
\hline $35-44$ & $9,131(75.5)$ & $1,252(10.4)$ & $1,712(14.2)$ & \\
\hline $45-54$ & $7,790(79.5$ & $780(8.0)$ & $1,230(12.6)$ & \\
\hline $54-65$ & $3,057(83.0)$ & $230(6.2)$ & $398(10.8)$ & \\
\hline $65+$ & $989(90.4)$ & $28(2.6)$ & $77(7.0)$ & \\
\hline \multicolumn{5}{|l|}{ Sex } \\
\hline Male & $27,729(76.5)$ & $3,783(10.4)$ & $4,718(13.0)$ & 2.0 \\
\hline Female & $14,069(71.44)$ & $2,284(11.6)$ & $3,341(17.0)$ & \\
\hline \multicolumn{5}{|l|}{ Geography } \\
\hline Southern urban & $35,242(81.8)$ & $3,878(9.0)$ & $3,979(9.23)$ & 0.6 \\
\hline Northern rural & $869(30.7)$ & $494(17.44)$ & $1,470(51.9)$ & \\
\hline Northern urban & $2,506(47.3)$ & $1,006(19.0)$ & $1,782(33.7)$ & \\
\hline Southern rural & $3,181(67.7)$ & $689(14.7)$ & $868(17.6)$ & \\
\hline \multicolumn{5}{|l|}{ Income } \\
\hline 1 (lowest) & $13,604(71.2)$ & $2,286(12.0)$ & $3,205(16.8)$ & 3.9 \\
\hline 2 & $9,540(76.3)$ & $1,284(10.3)$ & $1,675(13.4)$ & \\
\hline 3 & $7,535(75.6)$ & $1,078(10.8)$ & $1,359(13.6)$ & \\
\hline 4 & $6,303(77.9)$ & $812(10.0)$ & $977(12.1)$ & \\
\hline 5 & $4,816(76.9)$ & $607(9.7)$ & $843(13.5)$ & \\
\hline Deep tissue infections & $1,316(3.2))$ & $143(2.4)$ & $217(2.7)$ & 0.3 \\
\hline HIV status & $306(0.7)$ & $38(0.6)$ & $67(0.8)$ & 0.2 \\
\hline Mean days on methadone (SD)** & $376.8(471.6)$ & $385.6(454.7)$ & $444.9(490.3)$ & 16.4 \\
\hline Mean days on buprenorphine (SD)** & $56.6(169.5)$ & $40.9(136.7)$ & $29.0(107.3)$ & 16.3 \\
\hline
\end{tabular}

$d$, standardized difference; SD, standard deviation. ${ }^{* *}$ Subgroup analysis, $n=28,000$.

Continuous OAT (1-Year Treatment Retention)

We conducted a subgroup analysis of 1-year treatment retention in OAT using the ODB database only $(n=28,000)$. In a 5 -year prospective cohort study by the National Addiction Center in the United Kingdom, positive treatment outcomes were evident for patients after 1 year in OAT [28]. One-year treatment retention is a common measure used in several studies as a positive treatment outcome [6, 16, 29-33]. After their first treatment episode, all patients were followed to a maximum follow-up date of December 31,2016 . Continuous OAT (1-year treatment retention) was assessed based on prescription refill data (from the Ontario Drug Benefit database). Thirty days were chosen based on the interval used in previous research $[6,31,33]$. The database used for medication dispensing in this study might not capture doses administered in a hospital or provincial correctional settings. However, in Ontario, patients will typically continue to receive methadone or buprenorphine in these settings. Since most hospital admissions or provincial incarcerations are $<30$ days, this approach allows the analysis to be conducted without misinterpreting such events as treatment interruption.

Statistical Analysis

Descriptive statistics were calculated for all telemedicine utilization groups. Standardized differences were used to compare co- variates. Standardized differences over $10 \%$ were considered significant. For the main analysis, a Cox proportional hazard model was used to characterize the time to all-cause mortality event between the telemedicine patient groups. We then used a negative binomial regression model to calculate parameter estimates and odds ratios for the association between emergency department visits and hospitalizations and the outcomes. A subgroup analysis was conducted on Ontario Drug Benefit patients only to assess the 1 -year treatment retention variable. We adjusted for baseline covariates in the models, including sex, age, location of residence, income quintile, HIV status, and deep tissue infections. All data were analyzed using SAS Version 9.4 [34].

\section{Results}

Our main cohort included 55,924 patients on OAT. There were 41,798 patients $(74.7 \%)$ who received OAT predominantly by telemedicine, 6,067 patients (10.9\%) who received a mix of both in-person and telemedicine care, and 8,059 patients (14.4\%) patients who received OAT predominantly in person (Table 1). 
Table 2. Health system use and location of residence

\begin{tabular}{|c|c|c|c|c|c|c|}
\hline \multirow[t]{2}{*}{ Variable } & \multicolumn{2}{|c|}{ Predominantly in person } & \multicolumn{2}{|l|}{ Mixed } & \multicolumn{2}{|c|}{$\begin{array}{l}\text { Predominantly } \\
\text { telemedicine }\end{array}$} \\
\hline & $\begin{array}{l}\text { north, } \\
n=3,375\end{array}$ & $\begin{array}{l}\text { south, } \\
n=38,421\end{array}$ & $\begin{array}{l}\text { north, } \\
n=1,501\end{array}$ & $\begin{array}{l}\text { south, } \\
n=4,567\end{array}$ & $\begin{array}{l}\text { north, } \\
n=3,252\end{array}$ & $\begin{array}{l}\text { south, } \\
n=4,808\end{array}$ \\
\hline Mean primary care visits & $11.9(21.4)$ & $19.1(41.5)$ & $9.5(15.6)$ & $14.3(27.2)$ & $9.1(14.8)$ & $14.6(27.5)$ \\
\hline Mean number of emergency department visits & $21.5(28.9)$ & $18.5(28.8)$ & $27.7(30.3)$ & $22.7(29.0)$ & $32.3(36.0)$ & $23.0(27.9)$ \\
\hline Mean number of hospitalizations & $5.3(6.5)$ & $5.0(7.2)$ & $5.2(6.0)$ & $4.4(5.8)$ & $5.0(5.3)$ & $4.3(5.0)$ \\
\hline Mean days on buprenorphine* & $85.2(193.5)$ & $53.5(166.4)$ & $63.2(162.1)$ & $34.0(127.1)$ & $36.9(121.8)$ & $24.0(96.6)$ \\
\hline Mean days on methadone* & $307.7(434.4)$ & $384.3(475.0)$ & $322.9(412.6)$ & $405.5(465.7)$ & $437(471.4)$ & $449.7(502.2)$ \\
\hline
\end{tabular}

* Subgroup analysis, $n=28,000$.

Table 3. All-cause mortality, health system usage, and treatment retention in telemedicine groups

\begin{tabular}{|c|c|c|}
\hline & $\begin{array}{l}\text { Patients, } \\
n\end{array}$ & $\begin{array}{l}\text { Adjusted effect size } \\
(95 \% \text { CI })\end{array}$ \\
\hline \multicolumn{3}{|l|}{ All-cause mortality } \\
\hline Predominantly in person (reference groups) & 41,798 & \\
\hline Mixed & 6,097 & OR $1.0(0.9-1.1)$ \\
\hline Predominantly telemedicine & 8,059 & OR $0.9(0.8-1.0)$ \\
\hline \multicolumn{3}{|l|}{ Emergency department visits } \\
\hline Predominantly in person (reference groups) & 41,798 & \\
\hline Mixed & 6,097 & IRR $1.2(1.2-1.3)$ \\
\hline Predominantly telemedicine & 8,059 & IRR $1.4(1.4-1.5)$ \\
\hline \multicolumn{3}{|l|}{ Opioid-related emergency department visits } \\
\hline Predominantly in person (reference groups) & 41,798 & \\
\hline Mixed & 6,097 & IRR $1.0(0.7-1.2)$ \\
\hline Predominantly telemedicine & 8,059 & IRR $1.1(0.9-1.3)$ \\
\hline \multicolumn{3}{|l|}{ Mental health-related emergency department visits } \\
\hline Predominantly in person (reference groups) & 41,798 & \\
\hline Mixed & 6,097 & IRR $1.5(1.3-1.7)$ \\
\hline Predominantly telemedicine & 8,059 & IRR $1.4(1.3-1.6)$ \\
\hline \multicolumn{3}{|l|}{ Hospitalizations } \\
\hline Predominantly in person (reference groups) & 41,798 & \\
\hline Mixed & 6,097 & IRR $0.9(0.9-0.9)$ \\
\hline Predominantly telemedicine & 8,059 & IRR $0.9(0.9-1.0)$ \\
\hline \multicolumn{3}{|l|}{ Opioid-related hospitalizations } \\
\hline Predominantly in person (reference groups) & 41,798 & \\
\hline Mixed & 6,097 & IRR $1.2(0.9-1.8)$ \\
\hline Predominantly telemedicine & 8,059 & IRR $1.3(0.9-1.7)$ \\
\hline \multicolumn{3}{|l|}{ Mental health-related hospitalizations } \\
\hline Predominantly in person (reference groups) & 41,798 & \\
\hline Mixed & 6,097 & IRR $1.1(1.0-1.2)$ \\
\hline Predominantly telemedicine & 8,059 & IRR $1.2(1.1-1.3)$ \\
\hline \multicolumn{3}{|l|}{ One-year treatment retention (buprenorphine) ${ }^{* *}$} \\
\hline Predominantly in person (reference groups) & 20,862 & \\
\hline Mixed & 3,619 & HR $1.0(1.0-1.1)$ \\
\hline Predominantly telemedicine & 4,019 & HR $1.1(1.0-1.3)$ \\
\hline \multicolumn{3}{|l|}{ One-year treatment retention (methadone)** } \\
\hline Predominantly in person (reference groups) & 20,862 & \\
\hline Mixed & 3,619 & HR $1.1(1.0-1.1)$ \\
\hline Predominantly telemedicine & 4,019 & HR $1.0(1.0-1.3)$ \\
\hline
\end{tabular}

OR, odds ratio; HR, hazard ratio; IRR, incidence rate ratio. ${ }^{* *}$ Subgroup analysis $(n=28,000)$. 


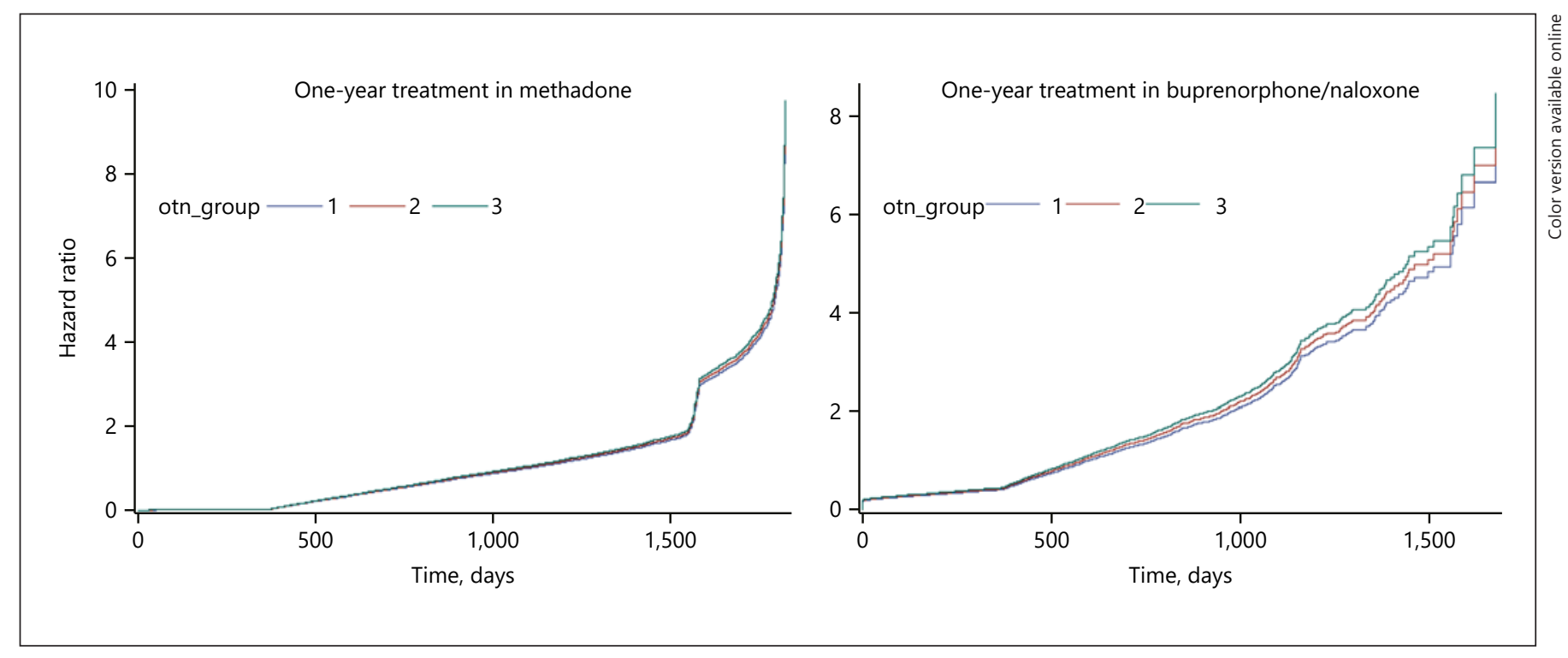

Fig. 2. Hazard ratio for patients in telemedicine groups in methadone maintenance and buprenorphine/naloxone maintenance treatment.

\section{Patient Characteristics}

Patient characteristics are presented in Table 2. There were no statistically significant differences between groups regarding the covariates, including sex, age, location of residence, or income. The records with missing information on the location of residence were deleted $(n=3)$. Over $30 \%$ of the cohort were in the lowest income quintile, and this was true in all 3 telemedicine patient groups. The records with missing information on the income quintile were reassigned to the lowest income group $(n=687,1.2 \%)$. The mean number of days patients were retained in methadone treatment was significantly higher among patients who received OAT predominantly by telemedicine (mean days retained in the in person = 376.8 , mixed $=385.6$, and telemedicine $=444.9$ ). However, the mean number of days patients were retained in buprenorphine treatment was significantly higher among patients who received OAT predominantly in person (mean days retained in the in-person group $=56.6$, mixed $=40.9$, and telemedicine $=29.0$ ).

\section{Health Service Usage by Location of Residence}

Health service usage is presented in Table 3. The mean number of primary care visits was higher among patients who received OAT predominantly in person $(p<0.001)$ and lower in Northern Ontario (mean primary care visits, north $=11.9$ and south $=19.1$ ). There were differences in the mean number of frequent emergency department vis- its across groups $(p<0.001)$, and notably, the mean number of emergency department visits was higher among patients in Northern Ontario compared to Southern Ontario. There were no statistically significant differences across telemedicine groups regarding the mean number of hospitalizations $(p=0.6858)$. However, the means were consistently higher across groups in Northern Ontario when compared to Southern Ontario.

\section{Association between Telemedicine, Treatment,}

Mortality, and Health Service Outcomes

We observed that receiving OAT predominantly by telemedicine was not associated with an increase in the likelihood of all-cause mortality when compared to OAT delivered in person ( $\mathrm{HR}=1.1,95 \% \mathrm{CI}: 1.0-1.1)$. The results are presented in Figure 2.

For the telemedicine group, the annual number of emergency department visits for reasons other than opioids and mental health increased by a factor of exponent $(\exp )(0.3562)=1.4,95 \%$ CI: $1.4-1.5$ (40\% increase). The annual number of mental health-related emergency department visits increased by a factor $\exp (0.0584)=1.5$, 95\% CI: $1.3-1.6$ (50\% increase). The annual number of mental health-related hospitalizations increased by a factor $\exp (0.0 .1721)=1.2,95 \%$ CI: $1.1-1.3$ (20\% increase), for each hospitalization in the in-person group.

However, receiving OAT by telemedicine was not associated with an increased likelihood of hospitalizations 
for reasons other than opioid or mental health factor exp $(-0.0716)=0.9,95 \%$ CI: 0.9-1.0, opioid-related emergency department visits factor $\exp (0.0584)=1.1,95 \% \mathrm{CI}$ : $0.9-1.3$, or opioid-related hospitalizations factor exp $(0.2368)=1.3,95 \%$ CI: $0.9-1.7$ compared to receiving OAT predominantly in person.

Receiving OAT predominantly by telemedicine was not associated with 1-year retention in $\mathrm{OAT}(\mathrm{OR}=1.0$, 95\% CI: 0.9-1.1) compared to patients receiving OAT in person in a subgroup of patients identified in the Ontario Drug Benefit Plan database. The results are presented in Table 3.

\section{Discussion}

The goal of this study was to evaluate telemedicine as a modality for OAT in Ontario, Canada, compared to inperson care. Our results align with other studies demonstrating that telemedicine-based OAT was associated with equal outcomes to in-person care regarding retention, opioid-related emergency department visits and hospitalizations, and mortality [16, 35-39]. However, we found that receiving OAT by telemedicine was associated with an increased likelihood of emergency department visits and hospitalizations for mental health reasons. There are 4 main findings derived from this study: (1) there was no significant association between OAT by telemedicine and the likelihood of all-cause mortality when compared to OAT delivered in person. (2) There was no significant association between OAT by telemedicine and opioid-related emergency department visits, hospitalizations, or hospitalizations for reasons other than opioids or mental health compared to OAT delivered in person. (3) Patients who accessed telemedicine-based OAT are just as likely to be retained for 1 year when compared to patients predominantly receiving in-person OAT. (4) Telemedicine-based OAT was associated with an increase in mental health-related emergency department visits and hospitalizations.

Our findings support previous literature indicating that OAT provided by telemedicine is equal to in-person care regarding mortality, opioid-related emergency department visit and hospitalizations, and 1-year treatment retention in OAT $[16,38]$. The findings are important because the use of telemedicine to deliver OAT has tremendous advantages in areas where access to care would be otherwise limited. Previous work has shown the median distance of a northern rural patient receiving treatment is over $100 \mathrm{~km}(60 \mathrm{mi})$ from the OAT provider [32]. Telemedicine can also in- crease access to care through programs such as ECHO (Extension for Community Healthcare Outcomes). ECHO uses telemedicine to build clinical capacity related to the treatment of opioid use disorder [40].

Concerning health service usage, several findings reported in this study may help health care resource planning in areas with similar OAT policies and programs. As we saw in this study, high emergency department rates have been linked to the lack of appropriate community health care services [41-43]. We noted that emergency department visits and hospitalizations were more elevated in the Northern and rural areas of Ontario. However, even after controlling for the location of residence of patients in our cohort, there was no significant association between OAT by telemedicine and opioid-related emergency department visits, hospitalizations, or hospitalizations for reasons other than opioids or mental health compared to OAT delivered in person. We did, however, find associations between mental health-related health service use and OAT. This study's results may indicate that telemedicine is mitigating the unnecessary use of acute services in Northern Ontario related to opioids. However, further research must be conducted to explore this finding further.

This study's subgroup analysis indicated that 1-year treatment retention was between 35 and $40 \%$ in our cohort. The retention in this cohort is crucial because it is much lower than retention rates represented in other studies (approximately 50\%) [16, 32, 44-46]. We attribute the lower retention to lower buprenorphine/naloxone retention in our study. For instance, the mean days patients were retained in buprenorphine in our cohort was between 35 and 60 days, compared to a mean of 350400 days retained in methadone. Mattick et al. [47] discuss several factors that contribute to lower retention in buprenorphine/naloxone, including that buprenorphine is a partial agonist and the risk of precipitated withdrawal in the first $24 \mathrm{~h}$. Methadone remains the most common treatment; however, we saw increased availability of buprenorphine-naloxone within the study period as an alternative to methadone [48]. It is important to note that only patients with ODB (public drug coverage) were included in the retention outcome analysis due to limited data availability. Patients with public drug coverage include seniors, youth under 25, persons with disabilities, and persons with high drug costs relative to their income. Therefore, they may experience barriers to accessing care that impact retention. Further study is needed to determine the association between such patient characteristics and treatment retention. 


\section{Limitations}

The main findings should be interpreted to indicate that OAT by telemedicine is equal to in-person care. However, we must acknowledge certain limitations within the study. Importantly, we cannot rule out confounding by unmeasured variables, resulting in a statistical finding of superior outcomes.

Other limitations are associated with the use of large databases. Administrative data are not originally intended to be used as a source of data for an academic study. Another significant limitation to this type of study is that variables related to quality of care, medication dosing, and psychological, societal, or economic factors are not available in the databases used. Therefore, they are not controlled for in the regression models, which may have led to residual confounding. Moreover, the data evaluated in this study only included people with opioid use disorder who had engaged in treatment at some point during the study period; therefore, the findings may not generalize to those with opioid use disorder who never involved in care. Nevertheless, the use of administrative health data was valuable for discovering trends and information regarding telemedicine usage, and to replicate the scope of this study's timeline and cohort using in-person techniques would be improbable and extremely difficult.

\section{Conclusion}

Our findings support the conclusion that telemedicine is equivalent to in-person care and a viable option for those seeking OAT. An opportunity to enhance convenience, reducing time spent traveling to and from the physician, and cost savings with respect to patient or physician travel are apparent benefits of telemedicine [13]. We recommend expanding telemedicine to enhance health human resources to treat opioid use disorder in areas where resources are limited.

\section{Acknowledgements}

We thank IC/ES Data Analytic Services and specifically Ryan $\mathrm{Ng}$ for his assistance with data extraction and database set up. We also thank the Patient and Family Advisory Committee members for sharing their stories and helping to guide the research project.

\section{Statement of Ethics}

Data were not directly collected from human subjects for this research. Written and informed consent was not needed for this study because we only used secondary data that are routinely collected from publicly funded health care organizations in Ontario. All data were collected in accordance with the Privacy Health Information Protection Act (PHIPA). We obtained ethical approval from the Laurentian University Ethics Board (File No. 6013835).

\section{Conflict of Interest Statement}

Dr. David Marsh maintains the following roles: Chief Medical Director at CATC (Canadian Addiction Treatment Center) and opioid agonist therapy provider. Dr. Marsh has no ownership stake in the CATC as a stipendiary employee. The authors do not foresee any conflict of interest as data will be made freely available to the public, and neither the Canadian Addiction Treatment Centers (CATC) nor the universities have the ability to prevent publication and dissemination of knowledge. The authors have no conflicts declared.

\section{Funding Sources}

We received funding from the Ontario Telemedicine Network (OTN) to conduct this research. OTN played a consultative role and reviewed the final manuscript.

\section{Author Contributions}

Kristen A. Morin: conceptualization, methodology, formal analysis, and writing - original draft. Matthew Parrotta: data curation, formal analysis, and writing - original draft preparation. Joseph K. Eibl: conceptualization, supervision, project administration, funding acquisition, and writing - review and editing. David C. Marsh: conceptualization, supervision, project administration, funding acquisition, and writing - review and editing.

\section{References}

1 Dhalla IA, Mamdani MM, Sivilotti ML, Kopp A, Qureshi O, Juurlink DN. Prescribing of opioid analgesics and related mortality before and after the introduction of long-acting oxycodone. CMAJ. 2009 Dec 8;181(12):891-6.

2 Lynas K. Ontario pharmacists concerned about the risks arising from approval of generic $\mathrm{Oxy}$ Contin. Can Pharm J. 2013 Jan;146(1):12-3.

3 Kiepek N, Groom B, Toppozini D, Kakekagumick K, Muileboom J, Kelly L. Evaluation of an inpatient medical withdrawal program in rural Ontario: a 1-year prospective study. Can J Rural Med. 2015;20(3):92-7.

4 Standing Committee on Health. Office of the speaker of the House of Commons. Ottawa, ON: Government of Canada; 2016.

5 Health Canada. Methadone maintenance therapy. Ottawa, ON; 2002.

6 Nosyk B, Marsh DC, Sun H, Schechter MT, Anis AH. Trends in methadone maintenance treatment participation, retention, and compliance to dosing guidelines in British Colum- bia, Canada: 1996-2006. J Subst Abuse Treat. $2010 \mathrm{Jul} ; 39(1): 22-31$.

7 Volkow ND, Frieden TR, Hyde PS, Cha SS Medication-assisted therapies - tackling the opioid-overdose epidemic. N Engl J Med. 2014 May 29;370(22):2063-6.

8 National consultation on the section 56 exemption requirement for methadone prescribing. Ottawa, ON: Health Canada; 2018.

9 Q\&As: methadone and diacetylmorphine. Toronto, ON: College of Nurses of Ontario; 2018.
Comparing In-Person and TelemedicineBased Opioid Agonist Treatment
Eur Addict Res 2021;27:268-276 DOI: $10.1159 / 000513471$ 
10 Regulations amending the narcotic control regulations and the new classes of practitioners regulations (diacetylmorphine (heroin) and methadone). Ottawa, ON: Canada Health; 2018.

11 Kiepek N, Hancock L, Toppozini D, Cromarty H, Morgan A, Kelly L. Facilitating medical withdrawal from opiates in rural Ontario. Rural Remote Health. 2012;12:2193.

12 Canadian Mental Health Association. Rural and Northern community issues in mental health. Ontario: Canadian Mental Health Association; 2009.

13 Brown EM. The Ontario telemedicine network: a case report. Telemed J E Health. 2013; 19:373-6.

14 Liddy C, Rowan MS, Afkham A, Maranger J, Keely E. Building access to specialist care through e-consultation. Open Med. 2013; $7(1): \mathrm{e} 1-8$.

15 O'Gorman LD, Hogenbirk JC. Driving distance to telemedicine units in Northern Ontario as a measure of potential access to healthcare. Telemed J E Health. 2016 Apr; 22(4):269-75.

16 Eibl JK, Gauthier G, Pellegrini D, Daiter J, Varenbut $\mathrm{M}$, Hogenbirk JC, et al. The effectiveness of telemedicine-delivered opioid agonist therapy in a supervised clinical setting. Drug Alcohol Depend. 2017 Jul 1;176:133-8.

17 ICES Data Dictionary. Institute for clinical and evaluative sciences data dictionary. 3.0.12 ed. Toronto, ON: ICES; 2016.

18 von Elm E, Altman DG, Egger M, Pocock SJ Gøtzsche PC, Vandenbroucke JP, et al. The strengthening the reporting of observational studies in epidemiology (STROBE) statement: guidelines for reporting observational studies. Rev Esp Salud Publica. 2008 MayJun;82(3):251-9.

19 Volkow ND. America's addiction to opioids: heroin and prescription drug abuse. In: National Institute on Drug Abuse. May 14, 2014

20 Termorshuizen F, Krol A, Prins M, Geskus R, van den Brink W, van Ameijden EJ. Prediction of relapse to frequent heroin use and the role of methadone prescription: an analysis of the Amsterdam Cohort Study among drug users. Drug Alcohol Depend. 2005 Aug 1; 79(2):231-40

21 Nosyk B, MacNab YC, Sun H, Fischer B, Marsh DC, Schechter MT, et al. Proportional hazards frailty models for recurrent methadone maintenance treatment. Am J Epidemiol. 2009 Sep 15;170(6):783-92.

22 Gomes T, Greaves S, Tadrous M, Mamdani MM, Paterson JM, Juurlink DN. Measuring the burden of opioid-related mortality in Ontario, Canada. J Addict Med. 2018 Sep/Oct;12: 418-9.
23 Gomes T GS, Martins D. Latest trends in opioid-related deaths in Ontario: 1991 to 2015. Toronto: Ontario Drug Policy Research Network; 2017.

24 Mamdani M, Rochon P, Juurlink DN, Anderson GM, Kopp A, Naglie G, et al. Effect of selective cyclooxygenase 2 inhibitors and naproxen on short-term risk of acute myocardial infarction in the elderly. Arch Intern Med. 2003 Feb 24;163(4):481-6.

25 Juurlink DN. Proton pump inhibitors and clopidogrel: putting the interaction in perspective. Circulation. 2009 Dec 08;120(23): $2310-2$.

26 Juurlink DN, Gomes T, Lipscombe LL, Austin PC, Hux JE, Mamdani MM. Adverse cardiovascular events during treatment with pioglitazone and rosiglitazone: population based cohort study. BMJ. 2009 Aug 18;339:b2942.

27 Measuring Up 2018. A yearly report on how Ontario's health system is performing. Chapter 5. Toronto, ON: Health Quality Ontario; 2018.

28 Gossop M, Marsden J, Stewart D, Kidd T. The National treatment outcome research study (NTORS): 4-5 year follow-up results. Addiction. 2003 Mar;98(3):291-303.

29 Sees KL, Delucchi KL, Masson C, Rosen A, Clark HW, Robillard H, et al. Methadone maintenance vs 180 -day psychosocially enriched detoxification for treatment of opioid dependence: a randomized controlled trial. JAMA. 2000 Mar 8;283(10):1303-10.

30 Weisner C, Matzger H, Kaskutas LA. How important is treatment? One-year outcomes of treated and untreated alcohol-dependent individuals. Addiction. 2003 Jul;98(7):90111.

31 Peles E, Linzy S, Kreek M, Adelson M. Oneyear and cumulative retention as predictors of success in methadone maintenance treatment: a comparison of two clinics in the United States and Israel. J Addict Dis. 2008;27(4): 11-25.

32 Eibl JK, Gomes T, Martins D, Camacho X, Juurlink DN, Mamdani MM, et al. Evaluating the effectiveness of first-time methadone maintenance therapy across Northern, rural, and urban regions of Ontario, Canada. J Addict Med. 2015 Dec;9(6):440-6.

33 Franklyn AM, Eibl JK, Gauthier G, Pellegrini D, Lightfoot NE, Marsh DC. The impact of benzodiazepine use in patients enrolled in opioid agonist therapy in Northern and rural Ontario. Harm Reduct J. 2017 Jan 26;14(1):6.

34 SAS version 9.4. In: System SA, editor. Cary, NC; 2020.

35 Ball C RA. The effectiveness of methadone maintenance treatment. New York: Springer Verlag; 1991.
36 Amato L, Davoli M, Perucci CA, Ferri M, Faggiano F, Mattick RP. An overview of systematic reviews of the effectiveness of opiate maintenance therapies: available evidence to inform clinical practice and research. J Subst Abuse Treat. 2005 Jun;28(4):321-9.

37 Bart G. Maintenance medication for opiate addiction: the foundation of recovery. J Addict Dis. 2012;31(3):207-25.

38 Young LB. Telemedicine interventions for substance-use disorder: a literature review. J Telemed Telecare. 2012 Jan;18(1):47-53.

39 Marsch LA, Guarino H, Acosta M, AponteMelendez Y, Cleland C, Grabinski M, et al. Web-based behavioral treatment for substance use disorders as a partial replacement of standard methadone maintenance treatment. J Subst Abuse Treat. 2014 Jan;46(1): 43-51.

40 Zurawski A, Komaromy M, Ceballos V, McAuley C, Arora S. Project ECHO brings innovation to community health worker training and support. J Health Care Poor Underserved. 2016;27(4A):53-61.

41 Genell Andren K, Rosenqvist U. Heavy users of an emergency department: a two year follow-up study. Soc Sci Med. 1987;25(7):82531.

42 Durbin J LE, Rush B. A study of hospital emergency service use, crisis service delivery and police response after mental health system enhancements. 2010.

43 Althaus F, Paroz S, Hugli O, Ghali WA, Daeppen JB, Peytremann-Bridevaux I, et al. Effectiveness of interventions targeting frequent users of emergency departments: a systematic review. Ann Emerg Med. 2011 Jul;58(1):41e42.

44 Gryczynski J, Mitchell SG, Jaffe JH, Kelly SM, Myers CP, O'Grady KE, et al. Retention in methadone and buprenorphine treatment among African Americans. J Subst Abuse Treat. 2013 Sep;45(3):287-92.

45 Hser YI, Saxon AJ, Huang D, Hasson A, Thomas C, Hillhouse M, et al. Treatment retention among patients randomized to buprenorphine/naloxone compared to methadone in a multi-site trial. Addiction. 2014 Jan; 109(1):79-87.

46 Mariolis T, Bosse J, Martin S, Wilson A, Chiodo L. A systematic review of the effectiveness of buprenorphine for opioid use disorder compared to other treatments: Implications for research and practice. J Addict Res Ther. 2019;10(2).

47 Mattick RP, Kimber J, Breen C, Davoli M. Buprenorphine maintenance versus placebo or methadone maintenance for opioid dependence. Cochrane Database Syst Rev. 2008 Apr;16(2):CD002207.

48 Strike C. A cross-Canada scan of methadone maintenance treatment policy developments. Center for Addiction and Mental Health and University of Toronto. 2011 